\title{
Performance Analysis of DWT-OFDM and FFT-OFDM using Various Digital Modulation Techniques and Channel Coding
}

\author{
Pratima Manhas \\ Research Scholar, FET, ECE \\ Manav Rachna International University,Fbd
}

\author{
M.K Soni, $\mathrm{PhD}$ \\ ED\& Dean, FET \\ Manav Rachna International University, Fbd
}

\begin{abstract}
Orthogonal frequency division multiplexing (OFDM) is a type of multicarrier modulation technique in which available bandwidth is divided into narrow bands at different frequencies. It is used to compress a large amount of data into a small amount of bandwidth. This is possible by dividing a large amount of data into smaller parts, then sending that data simultaneously over a number of frequencies. OFDM allows a large amount of data to be transmitted quickly and reliably, with a minimum of loss or interference. As OFDM is used in various applications like Wi-Fi, 4G, DAB \& WiMax, so its performance is an important factor and for this Bit Error ratio (BER) is calculated .This paper deals with the comparison between DWT based OFDM and FFT based OFDM for various digital modulation techniques and channel coding in terms of Bit error rate calculation. Simulation results shows that FFT based OFDM have better results as compared to DWT based OFDM.
\end{abstract}

\section{Keywords}

Bit error ratio (BER), FFT, DWT, orthogonal frequency division multiplexing (OFDM)

\section{INTRODUCTION}

OFDM is highly efficient technique in which high-rate data stream is split into a number of lower rate streams that are transmitted simultaneously over a number of sub-carriers. It removes the problem of multi-path channel fading effect, and has low spectrum efficiency. OFDM is used for both wired (ADSL/DSL) and wireless communication (Wireless OFDM is a combination of modulation and multiplexing. The paper is divided into following sections. Section 2 describes the
FFT/DWT based OFDM model .Simulation model and BER analysis is shown in section 3.Section 4 describes the simulation results and finally the conclusion is in section 5 .

\section{OFDM MODEL}

In OFDM transmitter, suppose the data bits are represented as $D=\{d 0, d 1, d 2, \ldots)$. Then there is a requirement of number of subcarriers which is used to send the given data. Lets assume that $\mathrm{N}$ subcarriers are used[1] . Each subcarriers are centred at frequencies that are orthogonal to each other (usually multiples of frequencies).After that any modulation techniques can be used (BPSK, QPSK \& QAM).

The data (D) has to be first converted from serial stream into parallel stream depending on the number of sub-carriers $(\mathrm{N})$. If there are $\mathrm{N}$ subcarriers allowed for the OFDM transmission, then the subcarriers are represented from 0 to $\mathrm{N}-1$. Now, the Serial to Parallel converter takes the serial stream of input bits and outputs $\mathrm{N}$ parallel streams (indexed from 0 to $\mathrm{N}-1$ ). These parallel streams are individually converted into the required digital modulation format (BPSK, QPSK, QAM etc..,). The outputs are represented as $\mathrm{S} 0, \mathrm{~S} 1, . . \mathrm{S}_{\mathrm{N}}$.. When the data bits are converted into the required modulation format, they need to be superimposed on the required orthogonal subcarriers for transmission. This is done by a series of NLAN).It is a bandwidth efficient technique but has certain limitations like high peak to average power ratio(PAPR), intercarrier interference(ICI).Parallel sinusoidal oscillators which is tuned to $\mathrm{N}$ orthogonal frequencies $\left(\mathrm{f}_{0}, \mathrm{f}_{1}, \ldots \mathrm{f}_{\mathrm{N}-1}\right)$. Finally, the resultantoutput from the $\mathrm{N}$ parallel arms are summed up together to produce the OFDM signal. The following Fig 1 illustrates the basic concept of OFDM transmission [2].

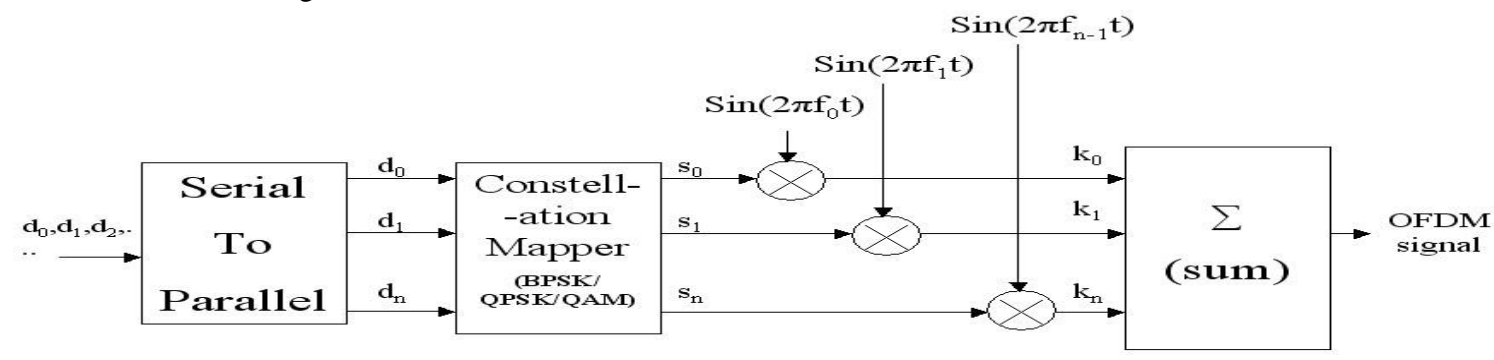

Fig 1: Generation of OFDM system 
Data $=1,1,0,0,1,0,0,1,0,1,1,1,0,0,1,1,1,0,1$

\begin{tabular}{|l|l|l|l|l|l|}
\hline Time & d0 & d1 & d2 & d3 & d4 \\
\hline $\mathrm{t} 1$ & 1 & 1 & 0 & 0 & 1 \\
\hline $\mathrm{t} 2$ & 0 & 0 & 1 & 0 & 1 \\
\hline $\mathrm{t} 3$ & 1 & 1 & 0 & 0 & 1 \\
\hline $\mathrm{t} 4$ & 1 & 1 & 1 & 0 & 1 \\
\hline
\end{tabular}

Then after converting serial data into parallel, the mapping is done .BPSK mapping is represented as

\begin{tabular}{|l|l|l|l|l|l|}
\hline Time & S0 & S1 & S2 & S3 & S4 \\
\hline $\mathrm{t} 1$ & 1 & 1 & -1 & -1 & 1 \\
\hline $\mathrm{t} 2$ & -1 & -1 & 1 & -1 & 1 \\
\hline $\mathrm{t} 3$ & 1 & 1 & -1 & -1 & 1 \\
\hline $\mathrm{t} 4$ & 1 & 1 & 1 & -1 & 1 \\
\hline
\end{tabular}

After BPSK mapping then mapping outputs is multiply by orthogonal frequency subcarriers. It is shown below:

\begin{tabular}{|l|l|l|l|l|l|}
\hline Time & S0 & S1 & S2 & S3 & \\
\hline $\mathrm{t} 1+\Delta$ & $1 \mathrm{x} \sin 2 \pi \mathrm{f} 0 \mathrm{t}$ & $1 \mathrm{x} \sin 2 \pi \mathrm{f} 1 \mathrm{t}$ & $-1 \mathrm{x} \sin 2 \pi \mathrm{f} 2 \mathrm{t}$ & $-1 \mathrm{x} \sin 2 \pi \mathrm{f} 3 \mathrm{t}$ & $1 \mathrm{x} \sin 2 \pi \mathrm{f} 4 \mathrm{t}$ \\
\hline $\mathrm{t} 2+\Delta$ & $-1 \mathrm{x} \sin 2 \pi \mathrm{f} 0 \mathrm{t}$ & $-1 \mathrm{x} \sin 2 \pi \mathrm{f} 1 \mathrm{t}$ & $1 \mathrm{x} \sin 2 \pi \mathrm{f} 2 \mathrm{t}$ & $-1 \mathrm{x} \sin 2 \pi \mathrm{f} 3 \mathrm{t}$ & $1 \mathrm{x} \sin 2 \pi \mathrm{f} 4 \mathrm{t}$ \\
\hline $\mathrm{t} 3+\Delta$ & $1 \mathrm{x} \sin 2 \pi \mathrm{f} 0 \mathrm{t}$ & $1 \mathrm{x} \sin 2 \pi \mathrm{f} 1 \mathrm{t}$ & $-1 \mathrm{x} \sin 2 \pi \mathrm{f} 2 \mathrm{t}$ & $-1 \mathrm{x} \sin 2 \pi \mathrm{f} 3 \mathrm{t}$ & $1 \mathrm{x} \sin 2 \pi \mathrm{f} 4 \mathrm{t}$ \\
\hline $\mathrm{t} 4+\Delta$ & $1 \mathrm{x} \sin 2 \pi \mathrm{f} 0 \mathrm{t}$ & $1 \mathrm{x} \sin 2 \pi \mathrm{f} 1 \mathrm{t}$ & $1 \mathrm{x} \sin 2 \pi \mathrm{f} 2 \mathrm{t}$ & $-1 \mathrm{x} \sin 2 \pi \mathrm{f} 3 \mathrm{t}$ & $1 \mathrm{x} \sin 2 \pi \mathrm{f} 4 \mathrm{t}$ \\
\hline
\end{tabular}

Then summation of S0, S1, S2, S3 and S4 at $\mathrm{t} 1+\Delta, \mathrm{t} 2+\Delta$, $\mathrm{t} 3+\Delta, \mathrm{t} 4+\Delta$ gives OFDM1, OFDM2, OFDM3 and OFDM 4

\section{DWT based OFDM system}

Wavelet transform are popular and used for the analysis and compression of signals and images. Discrete wavelet transform are applied to discrete data sets and produce discrete outputs[3].
Discrete wavelet transforms map data from the time domain (the original or input data vector) to the wavelet domain. The DWT based OFDM system is shown in the figure2.

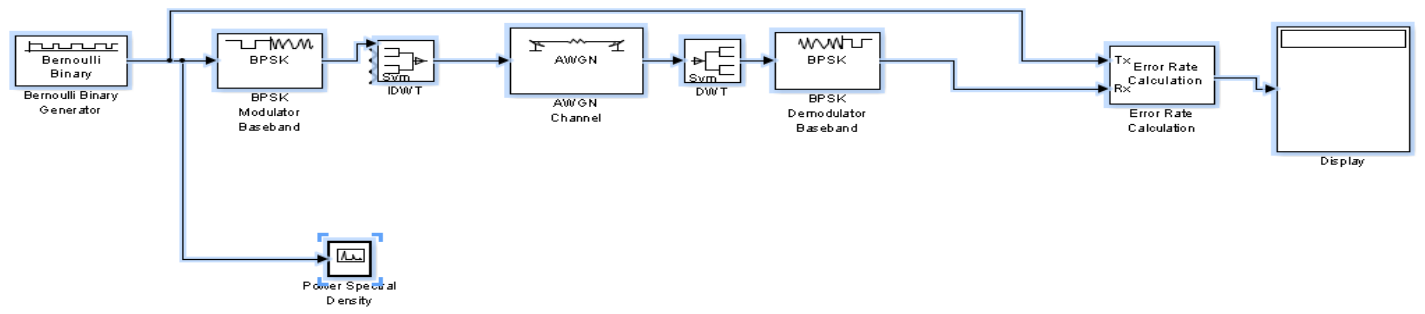

Fig 2: DWT based OFDM system using BPSK modulation

\section{FFT based OFDM system}

The fast Fourier transform (FFT) is a discrete Fourier transform algorithm which reduces the number of computations needed for $N$ points from $2 N^{2}$ to $2 N \lg N$.
An FFT is an algorithm that speeds up the calculation of a DFT [7]. The entire purpose of an FFT is to speed up the calculations. The FFT based OFDM system is shown in the fig 3 . 


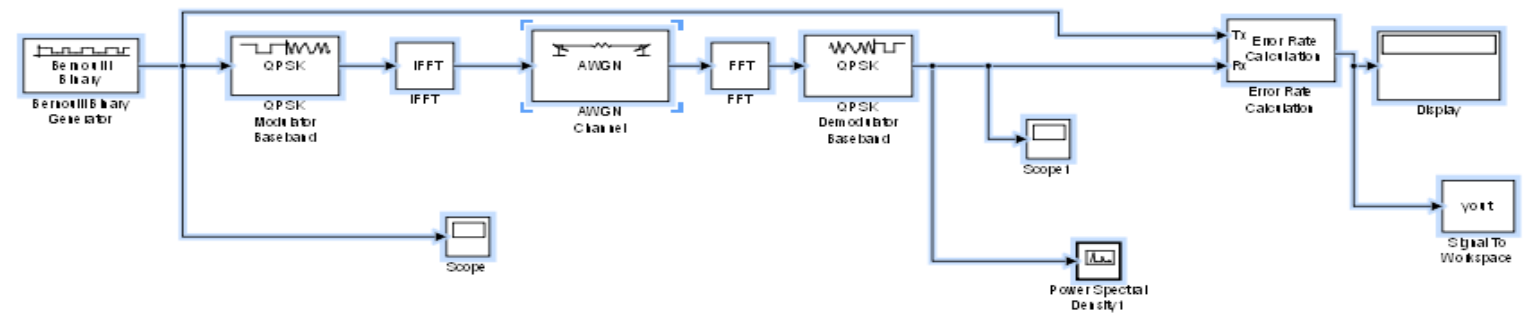

Fig 3: FFT based OFDM system using QPSK modulation

\section{SIMULATION MODEL}

Following steps are used to model OFDM system

1. Bernoulli binary generator is used to pass input signal. After passing the input signal then mapping is done using various modulations (BPSK/QPSK and QAM)

2. Then the mapped output is modelled using different transform (DWT/FFT)

The simulink model of DWT based OFDM \& FFT based OFDM system are shown below in Figures 4-9

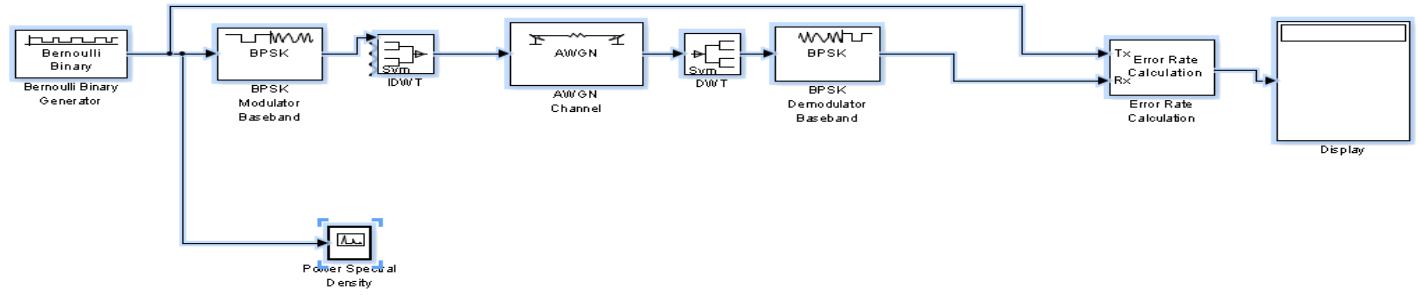

Fig 4: DWT based OFDM system using BPSK modulation

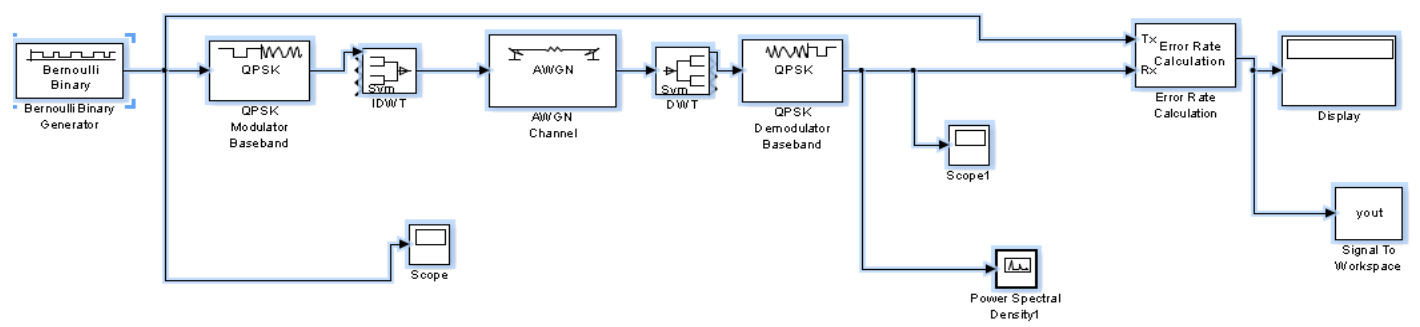

Fig 5: DWT based OFDM system using QPSK modulation

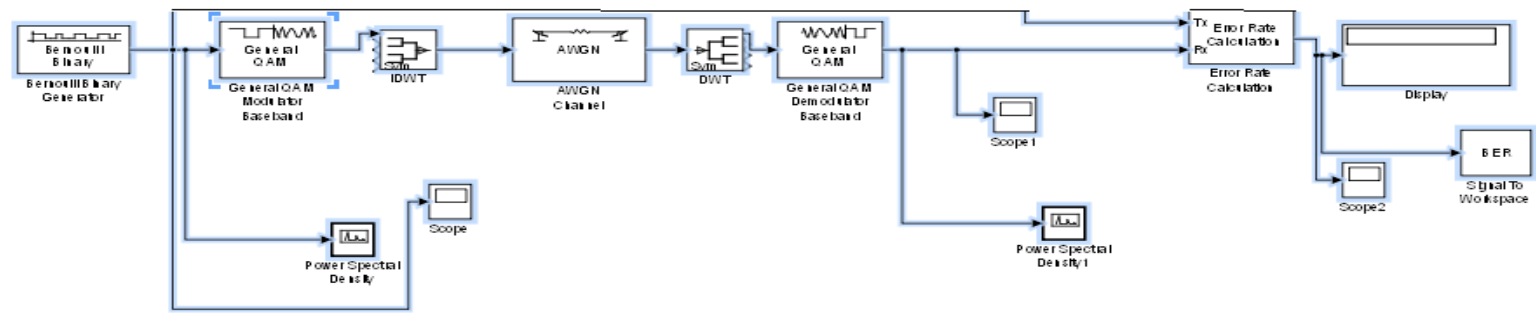

Fig 6: DWT based OFDM system using QAM modulation 


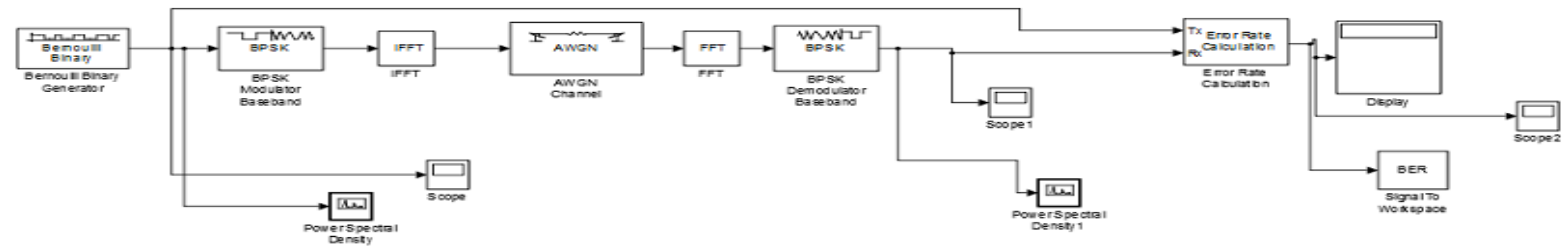

Fig 7: FFT based OFDM system using BPSK modulation

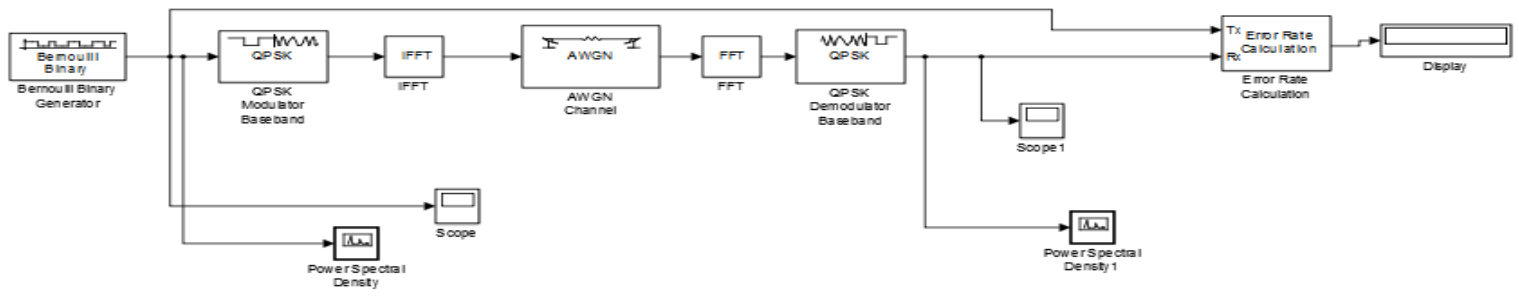

Fig 8: FFT based OFDM system using QPSK modulation

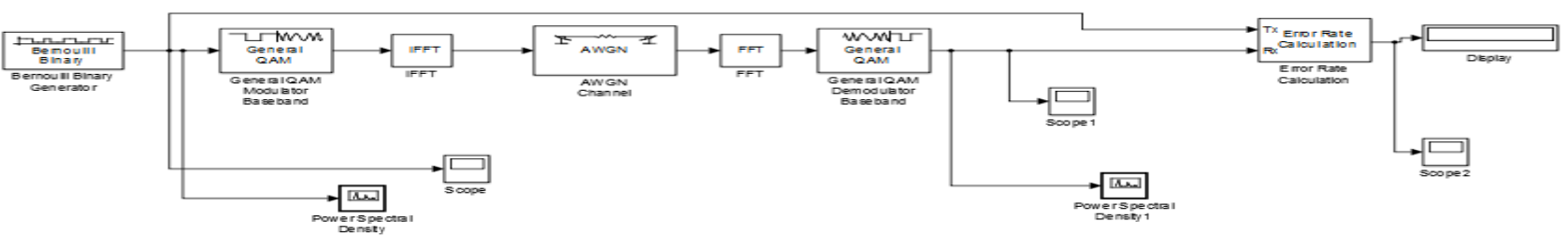

Fig 9: FFT based OFDM system using QAM modulation

BER Analysis of DWT-OFDM and FFT -OFDM for various modulation techniques
The BER results of DWT and FFT based OFDM system is shown in figures 10-15.

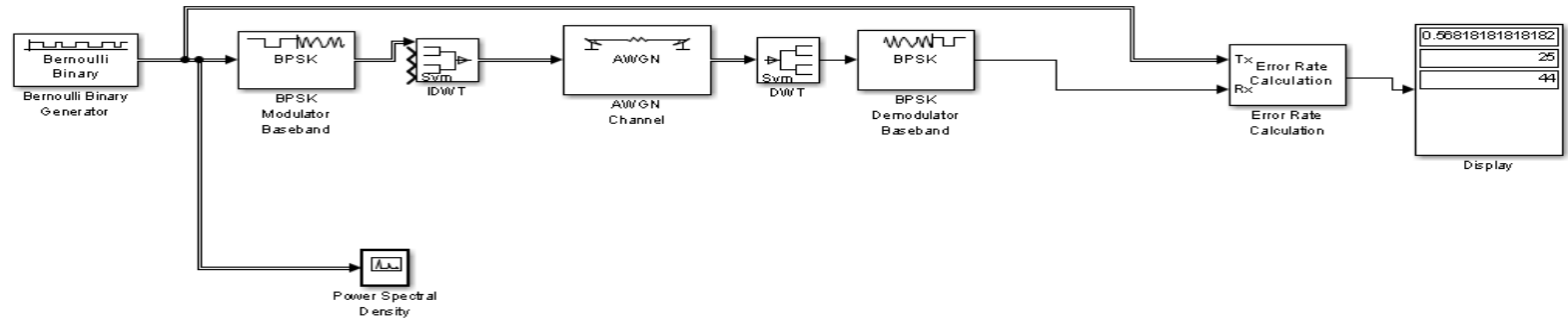

Fig 10: BER results of DWT based OFDM system using BPSK modulation

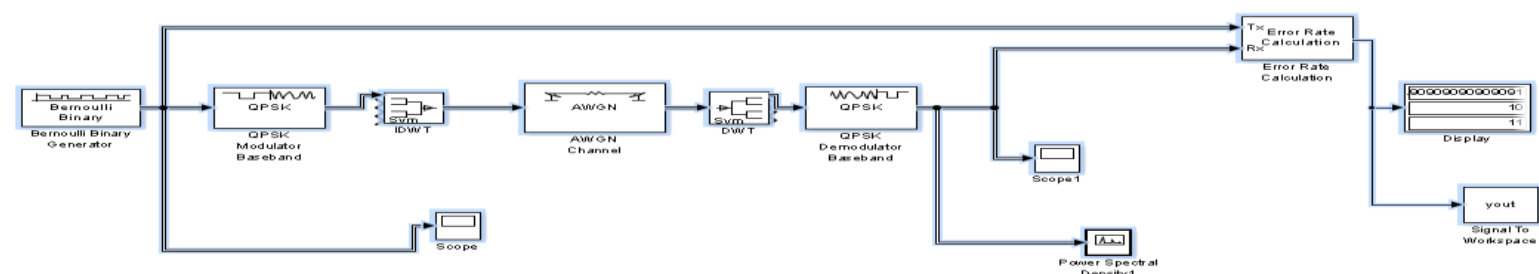

Fig 11: BER results of DWT based OFDM system using QPSK modulation 


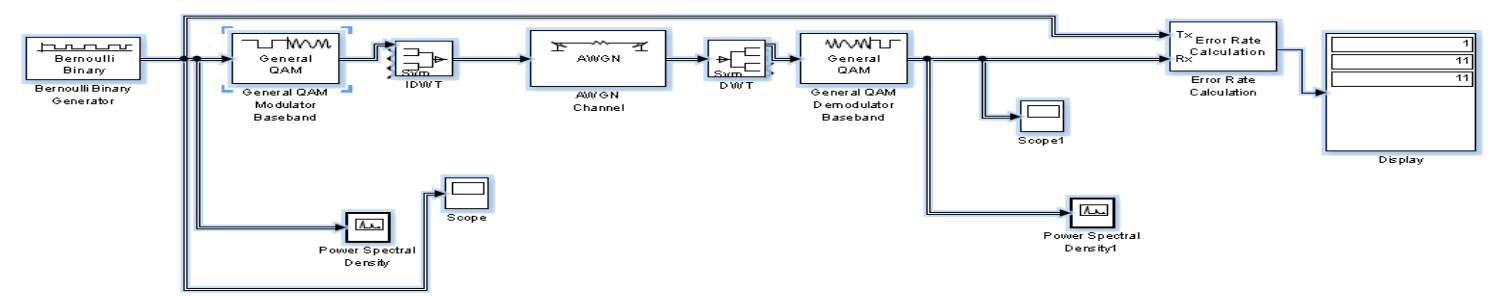

Fig 12: BER results of DWT based OFDM system using QAM modulation

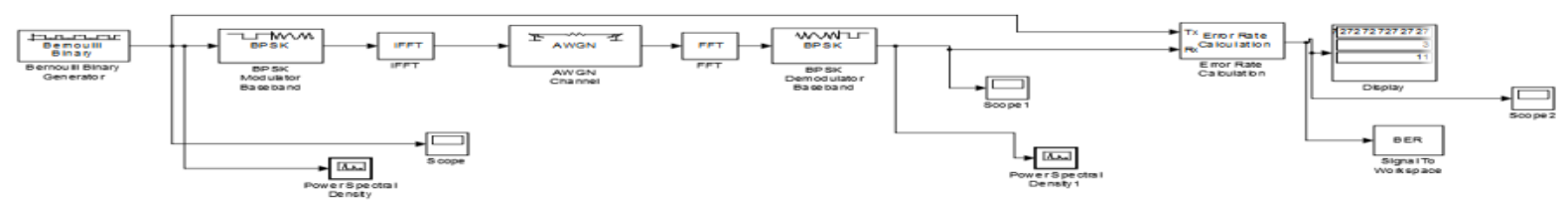

Fig 13: BER results of FFT based OFDM system using BPSK modulation

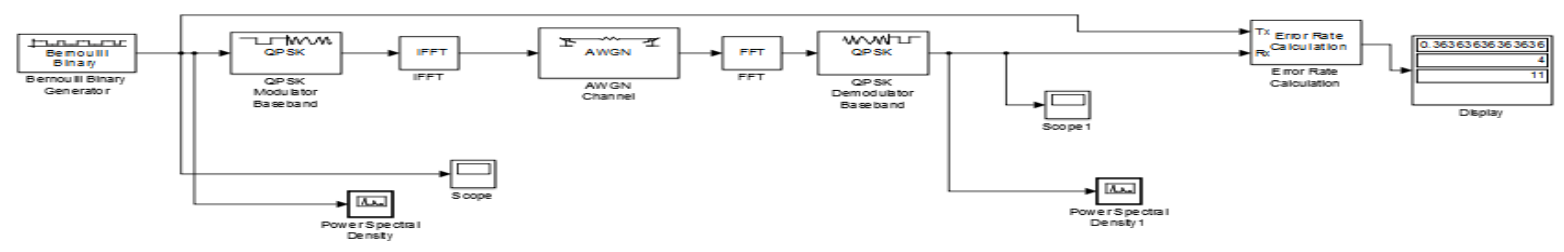

Fig 14: BER results of FFT based OFDM system using QPSK modulation

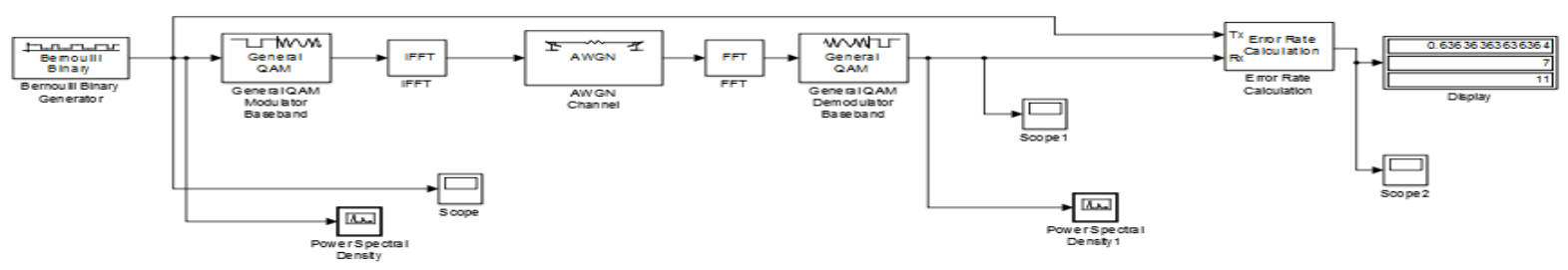

Fig 15: BER results of FFT based OFDM system using QAM modulation

\section{SIMULATION RESULTS}

The simulation parameters are given as

Modulation $=$ BPSK, QPSK and QAM

Channel $=$ AWGN

The BER calculation of both types of OFDM transform is shown below in table 1

Table 1. BER results of OFDM system using DWT/FFT transform

\begin{tabular}{|l|l|l|}
\hline $\begin{array}{l}\text { Transform based } \\
\text { OFDM system }\end{array}$ & Modulation & BER \\
\hline DWT & BPSK & 0.58 \\
\hline & QPSK & 0.909 \\
\hline & QAM & 1 \\
\hline FFT & BPSK & 0.27 \\
\hline
\end{tabular}

\begin{tabular}{|l|l|l|}
\hline & QPSK & 0.3636 \\
\hline & QAM & 0.636 \\
\hline
\end{tabular}

The table 1 compares the BER results using various modulations (BPSK, QPSK and QAM) along with different transform (DWT/FFT).Simulation results shows that using FFT transform along with BPSK modulation produces minimum BER. So in order to model OFDM system, FFT transform is used.

\section{CONCULSION}

OFDM is an emerging multi-carrier modulation scheme and multicarrier scheme supports high data rate. As the performance of OFDM depends upon the BER calculation .The simulation results shows that the FFT based OFDM has better BER value as compared to DWT based OFDM.So FFT based OFDM model with BPSK modulation can be used for transmission as it results in minimum BER as compared to others digital modulation techniques. The BER value can be further reduced by using channel coding methods with OFDM systems. 


\section{REFERENCES}

[1] Nee, Richard van, and Ramjee Prasad. OFDM for wireless multimedia communications. Artech House, Inc., 2000.

[2] Jha, Uma Shanker, and Ramjee Prasad. OFDM towards fixed and mobile broadband wireless access. Artech House, Inc., 2007.

[3] Liu, Hui, and Guoqing Li. OFDM-based broadband wireless networks: design and optimization. John Wiley \& Sons, 2005.

[4] Abdullah, Khaizuran, and Zahir M. Hussain. "Studies on dwt-ofdm and fft-ofdm systems." IEEE International Conference on Communication, Computer and Power. 2009.

[5] Saad, Waleed, et al. "An efficient technique for OFDM system using discrete wavelet transform." Advances in Grid and Pervasive Computing. Springer Berlin Heidelberg, 2010. 533-541.

[6] Abdullah, Khaizuran, Amin Z. Sadik, and Zahir M. Hussain. "On the dwt-and wpt-ofdm versus fft-ofdm."
GCC Conference \& Exhibition, 2009 5th IEEE. IEEE, 2009.

[7] Veena, M. B., and MN Shanmukha Swamy. "Performance analysis of DWT based OFDM over FFT based OFDM and implementing on FPGA." International Journal of VLSI Design \& Communication Systems (VLSICS) 2.3 (2011): 119-130.

[8] Gupta, Mahesh Kumar, and S. Tiwari. "Performance evaluation of conventional and wavelet based OFDM system." AEU-International Journal of Electronics and Communications 67.4 (2013): 348-354.

[9] Ooi, Andre Ken Lee, Micheal Drieberg, and Varun Jeoti. "DWT based FFT in practical OFDM systems." TENCON 2006. 2006 IEEE Region 10 Conference. IEEE, 2006.

[10] El-Hamid, Zahraa Abd, et al. "FFT/DWT/DCT OFDM channel estimation using EM algorithm in the presence of chaotic interleaving." Computer Engineering Conference (ICENCO), 2012 8th International. IEEE, 2012. 